\title{
3GPP LTE Downlink PHY Transceiver using Closed-loop Spatial Multiplexing in Frequency Selective Fading Environment
}

\author{
Darshana Kaushik \\ Dept. of ECE, DUIET \\ Dibrugarh University
}

\author{
Rituparna Borah \\ Dept. of ECE, DUIET \\ Dibrugarh University
}

\author{
Parismita Gogoi \\ Dept. of ECE, DUIET \\ Dibrugarh University
}

\begin{abstract}
LTE (Long Term Evolution) is a 3GPP (Third Generation Partnership Project) wireless standards that gives us wideband CDMA (Code Division Multiple Access) and HSPA (High Speed Packet Access) a third generation connectivity. LTE is the fourth generation (4G) of wireless communication technologies that brings the standard OFDMA (Orthogonal Frequency Division Multiple Access) modulation , MUMIMO (Multiuser Multiple Input Multiple Output) technology and different multipath fading model EVA (Extended Vehicular a), ETU (Extended Typical Urban) and EPA (Extended Pedestrian A) which allows the operator to use spectrum more efficiently to deliver high speed data. This paper characterizes the downlink performance of LTE. There are many metric to characterize the performance, but one of the most convenient and informative metric is the BER (Bit Error Rate). So the performance is characterized in terms of BER. In this paper the LTE system is modeled and simulated using MATLAB and the BER for $2 \times 2$ and $4 \times 4$ MIMO using QPSK and 16QAM modulation is obtained against different SNR values.
\end{abstract}

\section{General Terms}

Mobile Communication

\section{Keywords}

3GPP, LTE, MIMO, PHY

\section{INTRODUCTION}

LTE the next step in the evolution of the 3GPP, started in the year 2004 but it was first completed in the year 2008 as Release 8 of the 3GPP specification. It actually evolved from the earlier standards UMTS (Universal Mobile Telecommunications System) and HSPA (High Speed Packet Access) [1]. The Release 8 later evolved to Release 9 with some modification and then to Release 10, also known as LTE Advanced in the year 2011 by fulfilling the IMT (International Mobile Telecommunication) Advanced 4G requirements and Release 11 in the year 2012 by providing Advanced IP Interconnection of Services [2]. Currently work on Release 13 and Release 14 is being going on. The main aim behind LTE is that LTE provides a significant increase in bandwidth and better data services. LTE can operate in both the bands FDD (Frequency Division Duplex) and TDD (Time Division Duplex) of UMTS. LTE uses channel bandwidth of $1.4 \mathrm{MHz}-$ $20 \mathrm{MHz}$. The modulation schemes used in LTE are QPSK, 16QAM, 64QAM for the downlink whereas in the uplink 64QAM is optional for the handset to support and LTE uses OFDMA (Orthogonal Frequency Division Multiple Access) in the downlink and SC-FDMA (Single Carrier Frequency Division Multiple Access) in the uplink as multiple access schemes [3]. LTE supports the MIMO (Multiple Input Multiple Output) technology and MIMO is very essential for
LTE in order to support high data rates [4]. In downlink a wide choice of MIMO configuration is possible for LTE and there are different MIMO modes supported. For example there is transmit diversity, spatial multiplexing and cyclic delay diversity and a maximum of 4 antennas at the base station for LTE and maximum 8 antennas for LTE-A which is defined in the standards and a maximum of 4 receive antennas at the handset for LTE and maximum of 8 antennas for LTE-A [5]. In the uplink Multi-user collaborative MIMO is supported so several users can share the several resource block assignment. LTE uses a peak data rate of $150 \mathrm{Mbps}$ for $2 \times 2 \mathrm{MIMO}$ and 300 Mbps for $4 \times 4$ MIMO in the downlink and 75 Mbps in the uplink.

The network architecture of LTE is composed of E-UTRAN (Evolved Universal Terrestrial Radio Access Network) and the EPC (Evolved Packet Core). These parts were introduced in separate work item inside 3GPP. The work item responsible for E-UTRAN is the LTE and that for EPC is the SAE (System Architecture Evolution). It indicates that in this two work items the overall system architecture of LTE was evolved. The E-UTRAN which is the radio access network consists of only eNB's (eNodeB's) i.e. the base station, which means that a lot of functionality is concentrated inside the base station. The Physical layer, MAC (Medium Access Control) layer, PDCP (Packet Data Convergence Protocol) layer and RRC (Radio Resource Control) layer are terminating in the base station. The base stations the so called eNodeB's are directly connected to the core via the S1 interface. LTE does not have any radio network controller as in UMTS. So there is a very lean architecture of LTE. The base stations are connected to each other via the $\mathrm{X} 2$ interface. This is for example used to prepare handover situations and forward packets during handover. The EPC consists of different entities the MME (Mobility Management Entity), the Serving Gateway and the Packet Data Network Gateway which is the gateway to other IP (Internet Protocol) packets [2,6].

\section{THEORETICAL BACKGROUND}

In this paper focus is mainly on the downlink LTE so for the transmission of the data a better understanding of the LTE PHY radio interface is important. The radio interface covers the interface between the UE (User Equipment) and the network. The radio interface is composed of the Physical layer, Medium Access Control layer and the Radio Resource Control layer [3]. The downlink air interface technologies used in LTE are- OFDMA and MIMO. In OFDMA technique multiple simultaneous users are allocated to different subcarriers and the subcarrier spacing is $15 \mathrm{KHz}$ in the frequency domain. When there are 12 subcarriers in the time domain and 7 OFDMA symbols in the frequency domain with a subcarrier spacing of $15 \mathrm{KHz}$ it constitutes a resource block and several numbers of resource blocks combine together to form the resource grid. Thus in the time domain the entire band is 
divided into blocks of 12 subcarriers or $180 \mathrm{KHz}$ [6]. These blocks form the so called Physical Resource Blocks (PRB) .The subcarriers in these resource blocks are modulated using QPSK (Quadrature Phase Shift Keying) , 16QAM (16 Quadrature Amplitude Modulation), 64QAM (64 Quadrature Amplitude Modulation)

In QPSK modulation, the constellation diagram have four different positions and each modulation symbol are mapped to different positions in the constellation diagram. Thus QPSK needs 2 bits to encode different modulation symbols. 16QAM have 16 different positions in the constellation diagram so 4 bits are required to encode each modulation symbol. Similarly 64QAM have 64 different positions in the constellation diagram, so 6 bits are required to encode each modulation symbol. Higher order modulation i.e. 64QAM gives best result with strong signals and lower order modulation like QPSK gives best results with weak signals. As specified in the LTE standard the preferred channel coding techniques for LTE are Turbo Coding and Rate Matching [2]

In LTE standard MIMO technology is defined. In MIMO technology multiple antennas are used both at the transmitter and the receiver as a result MIMO wireless technology is able to considerably increase the capacity of a given channel. The throughput of the channel can also be linearly increased by increasing the number of transmit and receive antennas. Improvement in capacity and BER (Bit Error Rate) is seen by the use of MIMO. MIMO can actually be said to be a radio antenna technology as it uses multiple antennas at the transmitter and the receiver to carry the data. It is found that in MIMO between the transmitter and the receiver the signal can take many paths. The block diagram of a MIMO is shown in Figure $1[7,8]$. The channel with $\mathrm{N}$ outputs and $\mathrm{M}$ inputs is denoted as $\mathrm{M} \times \mathrm{N}$ matrix:

$$
H(n)=\left(\begin{array}{ccc}
h_{(n, 1,1)} & \cdots & h_{(n, 1, N)} \\
\vdots & \ddots & \vdots \\
h_{(n, M, 1)} & \cdots & h_{(n, M, N)}
\end{array}\right)
$$

In LTE standard four different types of MIMO algorithms are defined: receiver-combing, transmit diversity , beamforming and spatial-multiplexing.

In receiver-combining to improve the performance multiple versions of transmitted and received signals are combined. Two different types of combing methods are used at the receiver : MRC (Maximum Ratio Combining) and SC (Selection Combining). In MRC the transmitted signal is estimated by averaging the multiple received signal. In SC the transmitted signal is estimated by using only the received signal with the highest SNR (Signal-to-Noise Ratio) [5].

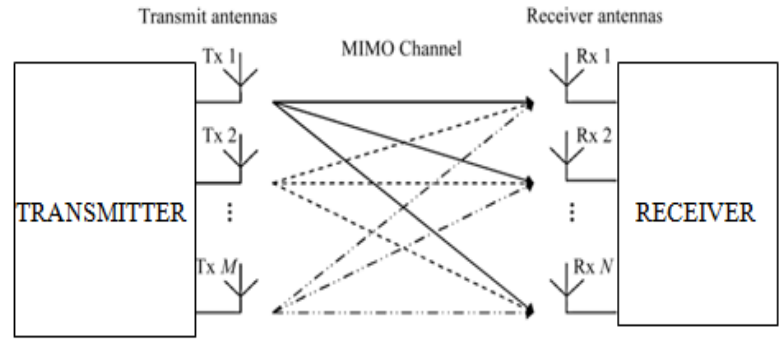

Figure 1: MIMO (Multiple Input Multiple Output)
In transmit diversity scheme different antennas transmit the information at each sub-carrier. Data rate is not increased by this scheme but it only makes the communication link more robust. Transmit diversity belongs to the class space-time coding. Space-time coding is used to extract as much information as possible from the received signal by combining all the copies of the received signal in an optimal way. The transmit-diversity scheme used in LTE is STBC (Space-Time Block Coding ). Space-time block codes are used to improve the reliability of data transfer across a number of antennas [2,5]. STBC uses both spatial and temporal diversity. STBC involves transmission of multiple copies of data. This helps to compensate for the channel problems such as fading and thermal noise. Although there is redundancy in the data some copies may arrive less corrupted at the receiver. The matrix representation of STBC is shown below-

$$
\left(\begin{array}{ccc}
S_{11} & \cdots & S_{1 n} \\
\vdots & \ddots & \vdots \\
S_{m 1} & \cdots & S_{m n}
\end{array}\right)
$$

Each row represents a time slot and each column represents one antenna's transmissions over time. Within the above matrix, $S_{m n}$ is the modulated symbol to be transmitted in time slot ' $m$ ' from antenna ' $n$ '.

Spatial multiplexing have the capability to transmit nonredundant information. As a result the data rate increases. Spatial multiplexing is susceptible to deficiencies in rank of the matrix representing the MIMO equation. Multiple techniques are introduced in LTE spatial multiplexing in order to minimize the probability of these rank deficiencies occurring and to harness its benefits. For $N_{t}$ transmit antennas and $N_{r}$ receive antennas, the maximum spatial multiplexing order is given by :-

$$
N_{s}=\min \left(N_{t}, N_{r}\right)
$$

here $N_{s}$ is the maximum spatial multiplexing order. MIMO Flat Fading Channel Matrix at sample time $\mathrm{n}$ is given by:-

$$
H(n)=\left(\begin{array}{ccc}
h_{(n, 1,1)} & \cdots & h_{(n, 1, N)} \\
\vdots & \ddots & \vdots \\
h_{(n, M, 1)} & \cdots & h_{(n, M, N)}
\end{array}\right)
$$

Here the time index $n=1, \cdots \cdots \cdots, n$ Samp, where $n$ Samp is the number of transmitted symbols in each subframe per antenna and $M, N$ are the number of transmitted and received antennas respectively $[2,5]$.

The channel matrix of MIMO multipath fading channel is

$$
H(n, k)=\left(\begin{array}{ccc}
h_{(n, k, 1,1)} & \cdots & h_{(n, k, 1, N)} \\
\vdots & \ddots & \vdots \\
h_{(n, k, M, 1)} & \cdots & h_{(n, k, M, N)}
\end{array}\right)
$$


Here the time index $n=1, \cdots \cdots \cdots, n$ Samp, where nSamp is the number of transmitted symbols in each subframe per antenna. The path-delay index $\mathrm{k}$ is $k=1 \cdots \cdots \cdots, L$, where $L$ the number of path delays.

In the 3GPP LTE standard three different multipath fading models are defined. They are EPA (Extended Pedestrian A), EVA (Extended Vehicular A), ETU (Extended Typical Urban). The delay profiles of these three models are shown in Table $1[2,9,10]$. In MIMO actually refers to sending and receiving multiple data on the same radio channel at the same time via multipath propagation and therefore there may be correlation between antenna port at both the transmitter and the receiver. It is always desirable to minimize this correlation. In LTE standard three different correlation level are specified and they are shown in Table 2 .

Table 1- Delay profiles for E-UTRAN channel models [9]

\begin{tabular}{|c|c|c|}
\hline Channel model & $\begin{array}{l}\text { Excess tap delay } \\
\text { (ns) }\end{array}$ & $\begin{array}{l}\text { Relative power } \\
\text { (dB) }\end{array}$ \\
\hline EPA & $\begin{array}{lll}{\left[\begin{array}{lllll}0 & 30 & 70 & 90 & 110 \\
190 & 410\end{array}\right]} & & \end{array}$ & 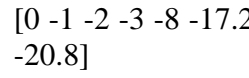 \\
\hline EVA & 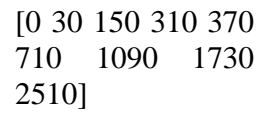 & 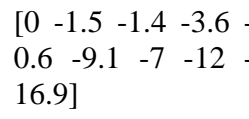 \\
\hline ETU & 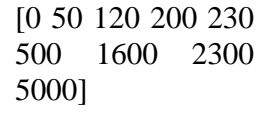 & $\begin{array}{l}{\left[\begin{array}{lllllll}-1 & -1 & -1 & 0 & 0 & 0 & -3 \\
5 & -7]\end{array}\right.} \\
\end{array}$ \\
\hline
\end{tabular}

Table 2- Correlation matrices for high, medium and low correlation [9]

\begin{tabular}{|l|l|l|}
\hline $\begin{array}{l}\text { LTE MIMO } \\
\text { channel correlation } \\
\text { levels }\end{array}$ & $\boldsymbol{\alpha}$ & $\boldsymbol{\beta}$ \\
\hline Low correlation & 0 & 0 \\
\hline Medium correlation & 0.3 & 0.9 \\
\hline High correlation & 0.9 & 0.9 \\
\hline
\end{tabular}

\section{SYSTEM MODEL}

The structure of the LTE downlink transceiver is shown in Figure 2 . It is composed of a transmitter, a channel model and a receiver. In LTE Physical layer which is interfaced by the MAC layer is connected by the transport channels. In the transmitter side the transport channel performs CRC (Cyclic Redundancy Code) generation, turbo coding based on $1 / 3$ rate and rate matching of the coded transport channel to handle any requested coding rates. The coded bits are then scrambled resulting in a block of scrambled bits, according to:-

$$
\tilde{b}^{(q)}(i)=\left(b^{(q)}(i)+c^{(q)}(i)\right) \bmod 2
$$

where $c^{(q)}(i)$ is the bock of scrambling sequence based on codeword q and $b^{(q)}(i)$ is the block of coded bits based on the codeword q. The blocks of scrambled bits are then modulated using modulation mapper, which produces complex valued modulation symbols:-

$$
x=I+j Q
$$

where I is the In-phase component and Q is the Quadrature components of the modulated symbol. The complex valued modulated symbols are mapped onto one or several layers :-

$$
x(i)=\left[x^{(0)}(i) \ldots \ldots \ldots \ldots . . . x^{(v-1)}(i)\right]^{T}
$$

where $i=0,1,2, \ldots \ldots \ldots . M_{\text {symb }}^{\text {layer }}-1, \mathrm{v}$ is the number of layers and $M_{\text {symb }}^{\text {layer }}$ is the number of modulation symbols per layer. The block of vectors from the layered mapping is then precoded using the precoder and produces an output:-

$$
\begin{aligned}
& y(i)=\left[\ldots \ldots \ldots \ldots y^{p}(i) \ldots \ldots \ldots \ldots\right]^{T} \\
& \text { where } \quad i=0,1,2, \ldots \ldots \ldots . M_{s y m b}^{a p}-1 \quad \text { and } y^{p}(i)
\end{aligned}
$$
represents the signal for each antenna port $\mathrm{p}$. To properly decode the data the terminal in LTE needs Reference Signals. After Precoding reference signals are generated, the coded bits are then mapped to the physical resource blocks. Then OFDM (Orthogonal Frequency Division Multiplexing) transmission is applied to the resource grid which produces the transmitted symbols.

The transmitted symbols are then passed through a MIMO fading channel which is distorted by AWGN noise.

After the symbols are passed through the channel it will be received at the receiver and in the receiver the symbols will be recovered based on the reverse operations as on the transmitter side.

The receiver will receive the OFDM signals processed by the channel, an estimate of the noise variance per received channel, the transmitted reference signals and demodulate the OFDM symbols to generate the best estimate of the transmitted symbols. 


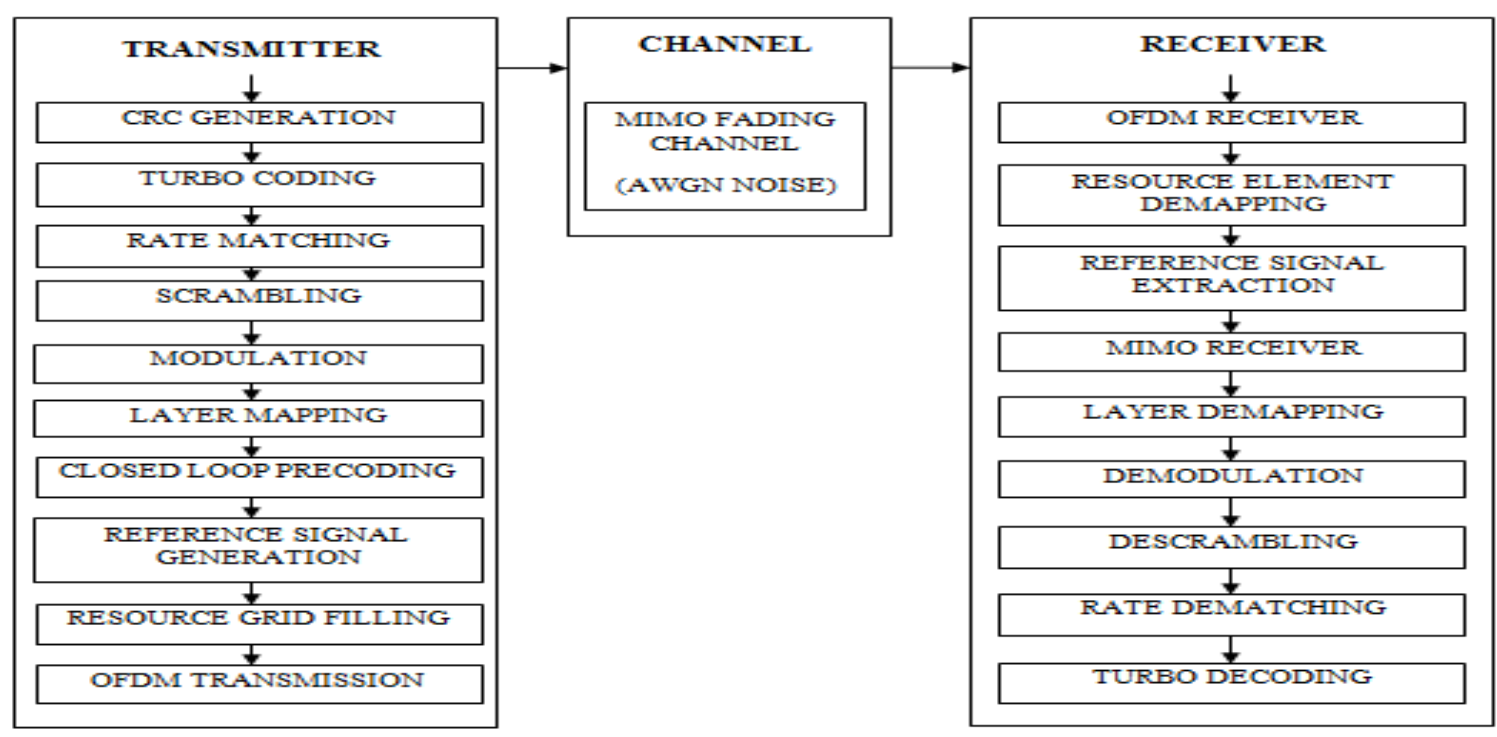

Figure 2: System Model of LTE Downlink

\section{EXPERIMENTAL RESULTS}

The information bits are generated in the transmitter side. The bits are than modulated using different modulation schemes such as QPSK and 16QAM. The modulated bits are transmitted using two/ four transmitting antennas and sent to the receiver through a MIMO Fading Channel and AWGN noise is added. The transmitted signal is received by two/four receiving antennas at the frontend of the receiver. It is then demodulated to get the desired signal. Finally the signal is sent to the user. Bit-error rate (BER) is plotted against different SNR (Signal to Noise ratio) values. The system has been developed using communication tool box available in MATLAB.

a) Parameters used

The parameters used are listed in Table 3, Table 4 and Table 5.

Table 3: Cell Width Configuration

\begin{tabular}{|c|c|}
\hline Parameters & Values \\
\hline No of Frames & 1024 \\
\hline No of resource blocks & 50 \\
\hline No of Transmit antennas & $2 / 4$ \\
& \\
\hline Cell ID & 0 \\
& \\
\hline Cyclic prefix & Normal (7) \\
& \\
\hline Duplex mode & FDD (Frequency Division \\
& Duplex) \\
\hline SNR Range & $0: 8$ (QPSK) \\
& $8: 13(16 \mathrm{Q}$ AM) \\
\hline
\end{tabular}

Table 4: Channel Model Configuration

\begin{tabular}{|c|c|}
\hline Parameters & Values \\
\hline No of received antennas & $2 / 4$ \\
\hline Downlink channel & $\begin{array}{c}\text { PDSCH (Physical } \\
\text { downlink shared channel) }\end{array}$ \\
\hline Channel bandwidth & $10 \mathrm{MHz}$ \\
\hline Channel model & Frequency selective \\
\hline Doppler shift & 5 \\
\hline Fading type & MIMO channel fading \\
\hline Noise type & AWGN \\
\hline MIMO correlation & Low \\
\hline Normalize path gain & On \\
\hline $\begin{array}{c}\text { Normalize for transmission } \\
\text { antennas }\end{array}$ & On \\
\hline
\end{tabular}

Table 5: Modulation and Equalization

\begin{tabular}{|c|c|}
\hline Parameters & Values \\
\hline Transmission mode & 4 (Spatial multiplexing) \\
\hline Modulation & QPSK/ 16QAM \\
\hline Mode type & QPSK/16QAM \\
\hline Coding rate & $1 / 3$ \\
\hline Equalization mode & MMSE \\
\hline Demodulation & QPSK/ 16QAM \\
\hline
\end{tabular}




\section{b) BER vs. SNR plots}

The BER is defined as the quality of the digital link which is calculated from the number of bits received with error divided by the number of bits transmitted by the transmitter and SNR is defined as the ratio of the received signal strength over the noise strength in the frequency range of the operation. SNR is inversely related to BER, that is high BER causes low SNR. High BER causes increase in delay and decreases throughput. SNR is used to study the quality of a communication link.

The BER vs. SNR plots for QPSK and 16QAM using LTE PHY transceiver model are shown in the Figure 3 and Figure 4 respectively. BER values for $2 \times 2$ and $4 \times 4$ MIMO for different values of SNR are listed for QPSK and 16QAM in Table 6 and Table 7 respectively.

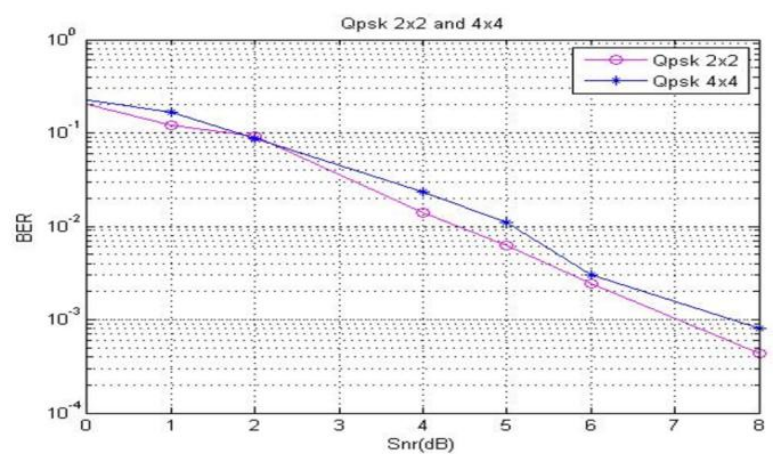

Figure 3: BER vs. SNR for $2 \times 2$ and $4 \times 4$ QPSK

Table 6: BER values for $2 \times 2$ and $4 \times 4$ QPSK

\begin{tabular}{|c|c|c|c|}
\hline $\begin{array}{c}\text { Sl. } \\
\text { No }\end{array}$ & Snr (dB) & BER( Qpsk 2×2) & $\begin{array}{c}\text { BER( Qpsk } \\
\mathbf{4 \times 4})\end{array}$ \\
\hline 1 & 0 & 0.202200 & 0.225100 \\
\hline 2 & 1 & 0.121300 & 0.168300 \\
\hline 3 & 2 & 0.091910 & 0.088150 \\
\hline 4 & 4 & 0.013900 & 0.023140 \\
\hline 5 & 5 & 0.006233 & 0.011020 \\
\hline 6 & 6 & 0.002416 & 0.003029 \\
\hline 7 & 8 & 0.0004297 & 0.000798 \\
\hline
\end{tabular}

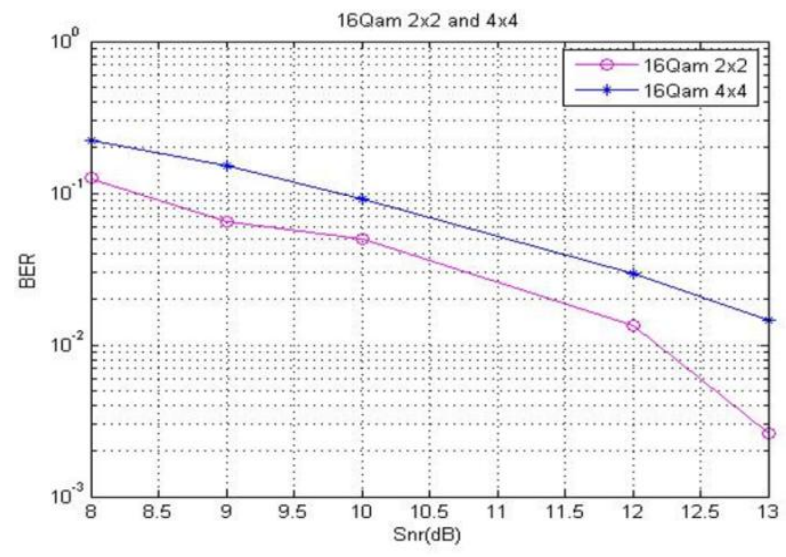

Figure 4: BER vs. SNR for $2 \times 2$ and $4 \times 4$ 16QAM
Table 7: BER values for $2 \times 2$ and $4 \times 4$ 16QAM

\begin{tabular}{|c|c|c|c|}
\hline SI.No & Snr (dB) & $\begin{array}{c}\text { BER( 16Qam } \\
\mathbf{2 \times 2}\end{array}$ & $\begin{array}{c}\text { BER( 16Qam } \\
\mathbf{4 \times 4})\end{array}$ \\
\hline 1 & 8 & 0.125000 & 0.222900 \\
\hline 2 & 9 & 0.064340 & 0.150000 \\
\hline 3 & 10 & 0.049850 & 0.090077 \\
\hline 4 & 12 & 0.013520 & 0.029490 \\
\hline 5 & 13 & 0.002624 & 0.014530 \\
\hline 6 & 14 & 0.002897 & 0.003359 \\
\hline
\end{tabular}

\section{CONCLUSION}

3GPP LTE which uses MIMO seems to be cornerstone due to the potential increase in data rate and transmit diversity offered by MIMO technology. In this paper the concept of 3GPP LTE has been demonstrated using the standard LTE model with transmission mode 4 with severely faded Frequency selective fading channel. BER curves play a very important role in the performance of the system. With the increasing value of SNR, the BER values must decrease for a better performance of the system. In this paper the BER vs. SNR for $2 \times 2$ and $4 \times 4$ MIMO using QPSK and 16QAM modulation and MMSE equalizer is plotted. It is seen that both for QPSK and 16QAM the BER curves for $2 \times 2$ is better with the MMSE equalizer.

Further the work can be extended using 64 QAM modulation and a better equalizer to get a better link performance between the transmitter and the receiver. In this paper although the Frequency selective fading channel is considered, several other severely faded channels can be used. The no of transmit and receive antennas can also be increased.

\section{REFERENCES}

[1] E. Dahlman , S. Parkvall , J. Skold , and P. Beming, 3G Evolution : HSDPA and LTE for Mobile Broadband, Academic Press, Jul. 2007.

[2] C. Cox, "An introduction to LTE, LTE-advanced, SAE VoLTE and 4G mobile communications", Second Edition, Wiley, 2014.

[3] 3GPP TS 36.201,"Evolved Universal Terrestrial Radio Access (E-UTRA); LTE Physical Layer General Description, version 8.3.0, Release 8.

[4] J. Zyren, "Overview of the 3GPP long term evolution physical layer", online, white paper, Freescale Semiconductor, July 2007.

[5] Juho. Lee, Jin-Kyu Han and Jianzhong (Charlie) Zhang ,"Review Article MIMO Technologies in 3GPP LTE and LTE-Advanced", Hindwai Publishing Corporation, EURASIP Journal on Wireless Communications and Networking, Volume 2009.

[6] 3GPP TS 36.300, " Evolved Universal Terrestrial Radio Access (E-UTRA) and Evolved Universal Terrestrial Radio Access Network (E-UTRAN); Overall description", version 8.9.0,Release 8.

[7] Aditya Kurve ," Multiuser MIMO systems: The future in the making", IEEE Potentials, December 2009. 
[8] T.S. Rappaport, "Wireless Communications-Principles and Practice", Pearson Education 1997.

[9] 3GPP TS 36.101, "Evolved Universal Terrestrial Radio Access (E-UTRA) UE (User Equipment) radio transmission and reception", version 10.3.0, Release 10.
[10] Jose A. del Peral-Rosado, Jose A. Lopez-Salcedo, Gonzalo Seco-Granados, Francesa Zanier, Massimo Crisci, "Evaluation of the LTE positioning capabilities under typical multipath channels", 6th Advanced Satellite Multimedia Systems Conference (ASMS) and 12th Signal Processing for Space Communications Workshop (SPSC), 2012 